Association for Information Systems

AIS Electronic Library (AISeL)

Wirtschaftsinformatik Proceedings 2001

Wirtschaftsinformatik

September 2001

\title{
Nachhaltigkeitsberichterstattung: Internet als Medium zur Integration der Geschäfts- und Umweltberichterstattung von Unternehmen
}

\author{
Ralf Isenmann \\ Universität Kaiserslautern, isenmann@bior.de \\ Christian Lenz \\ Universität Kaiserslautern, lenz@bior.de \\ Heiner Müller-Merbach \\ Universität Kaiserslautern, hmm@bior.de \\ Christian Reitz \\ dyneticsolutions.gmbh, reitz@dynetic.de
}

Follow this and additional works at: http://aisel.aisnet.org/wi2001

\section{Recommended Citation}

Isenmann, Ralf; Lenz, Christian; Müller-Merbach, Heiner; and Reitz, Christian, "Nachhaltigkeitsberichterstattung: Internet als Medium zur Integration der Geschäfts- und Umweltberichterstattung von Unternehmen" (2001). Wirtschaftsinformatik Proceedings 2001.58.

http://aisel.aisnet.org/wi2001/58

This material is brought to you by the Wirtschaftsinformatik at AIS Electronic Library (AISeL). It has been accepted for inclusion in Wirtschaftsinformatik Proceedings 2001 by an authorized administrator of AIS Electronic Library (AISeL). For more information, please contact elibrary@aisnet.org. 
In: Buhl, Hans Ulrich, u.a. (Hg.) 2001. Information Age Economy; 5. Internationale Tagung Wirtschaftsinformatik 2001. Heidelberg: Physica-Verlag

ISBN: 3-7908-1427-X

(C) Physica-Verlag Heidelberg 2001 


\title{
Nachhaltigkeitsberichterstattung: Internet als Medium zur Integration der Geschäfts- und Umweltberichterstattung von Unternehmen
}

\author{
Ralf Isenmann, Christian Lenz, Heiner Müller-Merbach \\ Universität Kaiserslautern
}

Christian Reitz

dynetic solutions gmbh

\begin{abstract}
Zusammenfassung: Das Internet wird als ein geeignetes Medium zur Integration der Geschäfts- und Umweltberichterstattung auf dem Weg zu einer Nachhaltigkeitsberichterstattung von Unternehmen behandelt. Es bietet den Unternehmen Unterstützungspotentiale in dreierlei Hinsicht: Erstens erleichtert es eine informative Offenlegung von Umweltinformation in Geschäfts- sowie von Geschäftsinformation in Umweltberichten. Zweitens ermöglicht es eine geschickte Verlinkung zwischen separaten Geschäfts- und Umweltberichten. Drittens eröffnet es eine effiziente Gestaltung von Nachhaltigkeitsberichten aus einem Guß. Insgesamt wird für den nutzenstiftenden Einsatz des Internet und die Ausschöpfung der damit einhergehenden medienspezifischen Unterstützungspotentiale als ein wichtiger Schritt zu einer Nachhaltigkeitsberichterstattung argumentiert.
\end{abstract}

Schlüsselworte: Geschäftsberichterstattung; Hypermedia, Internet; Nachhaltigkeitsberichterstattung, Umweltberichterstattung; XML; Zielgruppenorientierung

\section{Geschäfts- und Umweltberichterstattung}

Der Geschäfts- und Umweltbericht bilden die beiden Kerninstrumente der Geschäfts- und Umweltberichterstattung. Sie stehen bei der Vermittlung der ökonomischen und ökologischen Leistung von Unternehmen im Mittelpunkt.

Der Geschäftsbericht repräsentiert das in der Berichterstattungspraxis dominierende Instrument zur Präsentation für den Jahresabschluß von Unternehmen [Hütt00, S. 26], die offenlegungs- und publizitätspflichtig sind. Die Unternehmen vermitteln durch den Geschäftsbericht eine umfassende Übersicht über die wirtschaftliche Situation. Der Geschäftsbericht steht im Vordergrund der Marktkommunikation, er repräsentiert die ökonomische „Visitenkarte“ [BaKi97, S. 17] des Unternehmens v.a. für die Öffentlichkeit und dokumentiert die ökonomische Lei- 
stung insbesondere gegenüber den Investoren [Hütt00, S. 35; BaKi97, S. 21]. Der Geschäftsbericht besteht aus Sicht der Berichterstattungspraxis i.d.R. aus: (1) dem Jahresabschluß einschließlich Bilanz, Gewinn- und Verlustrechnung $(\mathrm{GuV})$ und Anhang, (2) dem Lagebericht sowie (3) Zusatzinformation z.B. zu einzelnen Geschäftsbereichen, Berichten von Vorstand und Aufsichtsrat, sozialen und Umweltaspekten sowie zur Unternehmensorganisation [KrGr99, S. 600].

Der Umweltbericht stellt in abgestufter Analogie zum Geschäftsbericht das zentrale Instrument in der Umweltkommunikation dar [IsWa99a, S. 1510]. Er repräsentiert eine zusammenhängende Publikation von Unternehmen und bietet den angesprochenen Zielgruppen, v.a. den Mitarbeitern und der Öffentlichkeit, zunehmend aber auch professionellen Nutzern wie z.B. Investoren und Finanzanalysten eine umfassende Übersicht mit quantifizierten Angaben über die geschäftsbedingten Umweltwirkungen einerseits sowie die ergriffenen und geplanten Umweltschutzaktivitäten andererseits [StScLe97, S. 9]. Umweltberichte dokumentieren die ökologische Leistung von Unternehmen [Stur00, S. 231]. Eine besondere Rolle in der Umweltberichterstattungspraxis spielen die „Umwelterklärungen“ nach der EG-Öko-Audit-Verordnung (EMAS). Sie stellen standortbezogene Umweltberichte dar, die die EMAS-Anforderungen erfüllen. Zahlenmäßig machen sie den überwiegenden Anteil der Umweltberichte in Deutschland aus [LoFi99, S. 4].

\section{Integrierte Geschäfts- und Umweltberichterstattung}

Eine integrierte Geschäfts- und Umweltberichterstattung kann nutzenstiftend sein, sowohl für die Unternehmen selbst wie auch für die jeweiligen Zielgruppen als die anvisierten Empfänger bzw. Nutzer von Geschäfts- und Umweltberichten [IsLeSc01, S. 49]. Die Unternehmen können erstens die Reichweite der Berichterstattung erhöhen und ggf. neue Zielgruppen ansprechen, zweitens den substantiellen Informationsgehalt vergrößern, drittens den Zielgruppen einen spezifischen Mehrwert bei der Berichterstattung bieten und viertens ihre unternehmerische Verantwortung für eine nachhaltige Entwicklung dokumentieren, indem sie über ökonomische und ökologische Aspekte berichten. Die Zielgruppen können ergänzend zum primär interessierenden Geschäfts- bzw. Umweltbericht erstens einen Einblick in die Verknüpfung zwischen der ökonomischen und ökologischen Leistung der Unternehmen erhalten. Zweitens wird ihnen eine detailliertere Informationsbasis für ihre beabsichtigten Zwecke geboten. Drittens können sie ihre Grundlage für eine umfassende Unternehmensbewertung z.B. für die Abschätzung von Geschäftsrisiken verbessern. Viertens werden sie für die wechselseitige Relevanz von Geschäfts- und Umweltschutzaktivitäten sensibilisiert.

Einerseits nimmt die Offenlegung von Umweltinformation in Geschäftsberichten durch eine gesonderte Ausweisung v.a. in Anhang und Lagebericht sowie in Bilanz und GuV seit den 1980er Jahren annähernd kontinuierlich zu [Uka86; 
Schu95; Dald99]. Vor allem bei Unternehmen in ökologischen Wettbewerbsfeldern wie z.B. Chemie, Pharma, Energie und Bergbau enthalten Geschäftsberichte inzwischen nahezu standardmäßig Umweltinformation. Diese ist zumeist in einem separaten kurzen Kapitel im Anhang oder Lagebericht zusammengefaßt und bietet einen ersten groben Überblick über Umweltmanagement, Umweltschutzinvestitionen und Umweltschutzaufwendungen [Schu95, S. 225; Dald99, S. 738]. Andererseits publizieren Unternehmen vermehrt ökonomische Effekte von Umweltschutzmaßnahmen in Umweltberichten [KPMG99, S. 25; LaAhDa01, S. 139, 166, 175]. Umweltschutz soll für Unternehmen wirtschaftlich sein. Zur Steuerung der betrieblichen Ressourcen ist es deshalb erforderlich, die Umweltschutzaktivitäten in monetären Größen zu erfassen, abzuschätzen und auszuweisen [Stur00, S. 116], zumal die einsetzbaren personellen und finanziellen Ressourcen begrenzt sind.

\section{Einsatz und Nutzen des Internet}

Der nutzenstiftende Einsatz des Internet zur Integration der Geschäfts- und Umweltberichterstattung wird hier detaillierter behandelt.

\subsection{Empirische Befunde}

Bei der Geschäftsberichterstattung gilt der Einsatz des Internet als nutzenstiftend [DAI00, S. 6; Helm98, S. 113]. Das Deutsche Aktieninstitut [DAI00, S. 5] stellt fest: „Moderne Investor-Relations-Arbeit ohne Unterstützung des Internet ist undenkbar“. Auch in den Agenturen zur Geschäftsberichterstellung erkennt man die Bedeutung des Internet [HGB01]. Gleichwohl mangelt es an der Qualität der Geschäftsberichterstattung [Anto00], so das Resümee zu neueren empirischen Befunden [DAI00; psyc00]: Die Mehrzahl der börsennotierten Unternehmen in Deutschland stellt den Geschäftsbericht ins Internet [DAI00, S. 39; Hü00, S. $\mathrm{XXX}$, auf eine mediengerechte Darbietung z.B. durch eine hypermediale Aufbereitung wird jedoch ebenso weitgehend verzichtet wie auf die Nutzung der Kommunikationsmöglichkeiten durch Dialoginstrumente. Die Anforderungen einer klaren Zielgruppenorientierung v.a. von privaten Investoren zur Offenlegung von Querbeziehungen - z.B. durch Hyperlinks innerhalb des Jahresabschlusses, aber auch eine geschickte Verlinkung im Lagebericht mit Information aus dem Shareholder-Value-Reporting [Helm98, S. 113] - werden kaum eingelöst.

Für die Umweltberichterstattung werden den eigenen Untersuchungen zufolge wie auch übereinstimmend mit anderen herangezogenen Studien verfügbare Hypermedia- und Internet-Technologien im Durchschnitt erst in geringem Maße eingesetzt [Isen01, S. 40; IsLeMM01, S. 103]: Von den veröffentlichten Umweltberichten in Deutschland sind lediglich rund 5\% im Internet verfügbar. Diese sind zumeist als ,eingestellte“, quasi als 1:1-Kopien von Umweltberichten in Printme- 
dien, bzw. als „angereicherte“ Umweltberichte zu klassifizieren [Isen01, S. 43]. Die erschließbaren inhalts- und darstellungs- [Isen01, S. 40] wie auch die zweckund prozeßbezogenen Unterstützungspotentiale [IsScMM01, S. 4] werden noch nicht ausgeschöpft. Über alle Kriterien hinweg ergeben sich beträchtliche Möglichkeiten zur Verbesserung. Dieser Gesamteindruck spiegelt sich im „Ranking 2000" wider [IÖfu00], in der die Umweltberichterstattungspraxis von deutschen Großunternehmen in Printmedien und im Internet untersucht wurde.

Die Möglichkeiten für eine Integration im Sinne einer ökonomische und ökologische Aspekte umfassenden Nachhaltigkeitsberichterstattung werden noch nicht ausgeschöpft. Hier bestehen zahlreiche Gestaltungschancen, Differenzierungsmöglichkeiten gegenüber Wettbewerbern und Profilierungsspielräume. Nach den vorliegenden Befunden aus wirtschaftszweigspezifischen und -übergreifenden empirischen Analysen [Jone99; Dald99; Schu95] wird auf gegenseitige Verweise zwischen Geschäfts- und Umweltberichten auch in Printmedien bislang weitgehend verzichtet. Ferner sind explizite Hinweise auf zusätzlich erstellte Umweltberichte wie auch auf deren Bezugsmöglichkeiten nur in wenigen Geschäftsberichten enthalten. Im Blick auf eine integrierte Geschäfts- und Umweltberichterstattung besteht für Unternehmen insgesamt noch erheblicher Nachholbedarf [Grön00, S. 199]. Dies gilt sowohl für traditionelle Publikationen und papiergebundene Berichte in Printmedien und erst recht für elektronische Publikationen in computergestützten Medien wie z.B. im Internet und auf CD-ROM.

Im Vergleich der technischen Standards und der Ausschöpfung der internetspezifischen Unterstützungspotentiale bei der Geschäfts- und Umweltberichterstattung im Internet für Deutschland ist eine Parallele festzustellen: Nach den neueren empirischen Befunden sind zum einen zwar beträchtliche Fortschritte festzustellen. Zum anderen besteht aber offensichtlich noch ein erheblicher Nachholbedarf, v.a. in puncto hypermedialer Darstellung, Nutzerfreundlichkeit, Zielgruppenorientierung und erweiterter Kommunikation. Die internetspezifischen Unterstützungspotentiale wie z.B. Recherche- und Suchfunktionen, Interaktivität, Dialog, Download und Hypermedialität werden insgesamt selten genutzt. Eine differenzierte Zielgruppenorientierung, Individualisierung und Personalisierung von Geschäftswie Umweltberichten ist nicht realisiert [Isen01, S. 44].

\subsection{Neuere Entwicklungstendenzen}

Vor dem Hintergrund der empirischen Befunde lassen sich die neueren Entwicklungstendenzen in der Geschäfts- und Umweltberichterstattung in vier charakteristische Bereiche bündeln [IsLeSc01, S. 52]: (1) Anstieg der Berichtszwecke: Die Anzeichen eines „Greening of Investor Relations“ sowie einer Dialogorientierung belegen zum einen die Relevanz der Umweltberichterstattung für professionelle Nutzer auf den Kapitalmärkten als aussagekräftige Informationsquelle zur Bewertung der Geschäftsentwicklung, v.a. zu Bonitätsprüfungen, Risiko- und Wett- 
bewerbsanalysen. Zum anderen setzen Unternehmen ihre Geschäfts- und Umweltberichte vermehrt als Instrumente zur aktiven Kommunikation mit Zielgruppen ein und beginnen, die technischen Möglichkeiten des Internet zum computergestützten Dialog zu nutzen. (2) Prozeßbezogene Unterstützung: Der Einsatz von computergestützten Medien und (Online-)Redaktionssystemen sowie die Möglichkeiten des crossmedialen Publizierens und von Push- und Pulltechnologien auf der einheitlichen Basis des Internet bieten den Unternehmen eine Reihe von prozessualen Unterstützungspotentialen [IsWa99a, S. 1513-1515]. Diese beziehen sich vorrangig auf eine rationelle Erstellung, effiziente Verwaltung, zielgruppenorientierte Verteilung und ansprechende Präsentation von Geschäfts- und Umweltberichten. (3) Inhaltliche Erweiterung: Der thematische Einbezug im Sinne einer Offenlegung von Umweltinformation in Geschäfts- sowie von Geschäftsinformation in Umweltberichten einerseits und die Initiativen zur Nachhaltigkeitsberichterstattung andererseits kennzeichnen die Tendenz zur umfassenden und integrierten Berichterstattung. Unternehmen beginnen, die ökonomischen, ökologischen, sozialen und humanen Aspekte ihrer Geschäftstätigkeit zu integrieren und z.T. einen einheitlichen Nachhaltigkeitsbericht zu publizieren. (4) Differenzierte Präsentation: Die Tendenzen zu Zielgruppenorientierung, hypermedialer Aufbereitung wie auch zur emotionalen Ansprache von Zielgruppen z.B. durch „Info-“ bzw. „Ecotainment“ sind der differenzierten Präsentation zuzuordnen. Einige proaktive Unternehmen [Henk00] nutzen bereits verschiedene Instrumente zur Berichterstattung, abgestimmt auf den Informationsbedarf, Detaillierungsgrad und Kommunikationskanal. Insgesamt sprechen die neueren Entwicklungstendenzen in der Geschäfts- und Umweltberichterstattung für eine integrierte Dokumentation der ökonomischen und ökologischen Leistung von Unternehmen.

\subsection{Unterstützungspotentiale des Internet}

Die Gestaltungschancen einer integrierten Geschäfts- und Umweltberichterstattung durch den Einsatz des Internet basieren auf den gestiegenen technischen Möglichkeiten moderner IKT [IsBu00, S. 137]. Diese eröffnen den Unternehmen eine Reihe von Unterstützungspotentialen. Sie ermöglichen eine geschickte Integration von Teilbereichen im Berichtswesen und bieten damit große Chancen für eine Unternehmenskommunikation mit Profil. Die erschließbaren Unterstützungspotentiale sind geradezu eine Herausforderung, diese für eine integrierte, effizient durchzuführende, hypermedial aufbereitete, auf den heterogenen Informationsbedarf von verschiedenen Zielgruppen abgestimmte und auf Dialog hin orientierte Geschäfts- und Umweltberichterstattung auszuschöpfen [IsWa99a, S. 1512]. Als Systematik dient eine vierteilige Gliederung [IsLeMM01, S. 100] (Abb. 1). Sie basiert methodisch auf zwei Heuristiken, dem Morphologischen Kasten und den Vier Causae von Aristoteles [IsLe01b, S. 204], und ist empirisch gegengeprüft. 


\begin{tabular}{|c|c|c|c|c|c|}
\hline $\begin{array}{l}\text { Zweckbezogene Unter- } \\
\text { stützungspotentiale }\end{array}$ & $\begin{array}{l}\text { Steuerung von } \\
\text { Ressourcen }\end{array}$ & $\begin{array}{l}\text { Information für } \\
\text { Zielgruppen }\end{array}$ & $\begin{array}{l}\text { Kommunikation mit } \\
\text { Zielgruppen }\end{array}$ & $\begin{array}{l}\text { Transaktion mit } \\
\text { Zielgruppen }\end{array}$ & $\cdots$ \\
\hline \multirow{2}{*}{$\begin{array}{l}\text { Prozeßbezogene Unter- } \\
\text { stützungspotentiale }\end{array}$} & \multicolumn{2}{|c|}{ Rationalisierung } & \multicolumn{2}{|c|}{ Zielgruppenorientierung } & $\ldots$ \\
\hline & \begin{tabular}{|c|} 
Verwaltung von \\
Berichtsbestandteilen
\end{tabular} & $\begin{array}{l}\text { Erstellung von } \\
\text { Berichten }\end{array}$ & $\begin{array}{l}\text { Verteilung von } \\
\text { Berichten }\end{array}$ & $\begin{array}{c}\text { Präsentation von } \\
\text { Berichten }\end{array}$ & $\cdots$ \\
\hline \multirow{2}{*}{$\begin{array}{l}\text { Inhaltsbezogene Unter- } \\
\text { stützungspotentiale }\end{array}$} & \multicolumn{2}{|c|}{$\begin{array}{l}\text { Geschäfts-/Umweltbericht } \\
\text { Nachhaltigkeitsbericht }\end{array}$} & \multicolumn{2}{|c|}{ Zusatzinformation } & $\ldots$ \\
\hline & $\begin{array}{c}\text { Zielgruppen- } \\
\text { spezifische Auswahl }\end{array}$ & $\begin{array}{c}\text { Themenauswahl, } \\
\text { Berichtsarchiv }\end{array}$ & $\begin{array}{l}\text { intern: } z . B \text {. } \\
\text { Umweltabteilung }\end{array}$ & \begin{tabular}{|} 
extern: Börsenkurs, \\
Ranking-Ergebnis
\end{tabular} & $\cdots$ \\
\hline $\begin{array}{l}\text { Darstellungsbezogene } \\
\text { Unterstützungspoten- } \\
\text { tiale }\end{array}$ & $\begin{array}{l}\text { Online-/Offline- } \\
\text { Verfügbarkeit }\end{array}$ & Nutzerführung & Hypermedialität & $\begin{array}{c}\text { Erweiterte } \\
\text { Kommunikation }\end{array}$ & $\cdots$ \\
\hline
\end{tabular}

Abbildung 1: Unterstützungspotentiale des Internet zur integrierten Berichterstattung

Die zweck- und prozeßbezogenen Unterstützungspotentiale umfassen u.a. eine effiziente Erstellung, Verwaltung und Verteilung von Geschäfts- und Umweltberichten. Sie repräsentieren die Unternehmenssicht und zielen primär auf eine Rationalisierung in der Berichterstattung. Die inhalts- und darstellungsbezogenen Unterstützungspotentiale beziehen sich überwiegend auf die Nutzersicht der Zielgruppen. Sie sind auf die in der Nachhaltigkeitsberichterstattung angelegte integrierte Dokumentation der ökonomischen und ökologischen Leistung der Unternehmen ausgelegt. Die vorgeschlagene Systematik von zweck-, prozeß-, inhaltsund darstellungsbezogenen Unterstützungspotentialen des Internet dient den Unternehmen als Strukturierungshilfe. Sie bietet eine systematische Übersicht für den nutzenstiftenden Einsatz des Internet zur integrierten Berichterstattung. Für die integrierte Geschäfts- und Umweltberichterstattung lassen sich die Unterstützungspotentiale hier in drei Stufen bündeln:

Stufe 1: Das Internet erleichtert eine informative Offenlegung von Umweltinformation in Geschäfts- (a) und von Geschäftsinformation in Umweltberichten (b). Sie wird v.a. durch die effiziente Verwaltung, die rationelle Erstellung von Berichten sowie deren kostengünstige Verteilung und ansprechende Präsentation unterstützt. Stufe 2: Ferner ermöglicht das Internet eine geschickte Verlinkung zwischen separaten Geschäfts- und Umweltberichten. Hierfür eigenen sich bspw. der Einsatz von Hyperlinks, unterstützender Nutzerführung und hilfreichen externen Verzweigungen z.B. zu Börsenkursen und Ranking-Organisationen. Stufe 3: Weiters eröffnet das Internet eine effiziente und zielgruppenorientierte Gestaltung von Nachhaltigkeitsberichten aus einem Guß.

Insgesamt kann das Internet als ein geeignetes Medium zur integrierten Geschäftsund Umweltberichterstattung dienen. Es bietet je nach präferierter Stufe der Integration große Unterstützungspotentiale im Sinne einer aussagekräftigen Offenlegung von Geschäfts- und Umweltinformation. Unternehmen können im Vergleich zu Printmedien einen Mehrwert bieten, indem sie die Berichte durch Zusatzlei- 
stungen wie erweiterte Kommunikation, hypermediale Darstellung, Interaktion, Dialog und Zielgruppenorientierung aufwerten [IsWa99a, S. 1513].

\subsection{Integrierte Berichterstattung mit XML}

Einen wichtigen Beitrag zur Ausschöpfung der internetspezifischen Unterstützungspotentiale zur Berichterstattung leistet der Einsatz von XML [LeIsRe01, S. 59]. Er ermöglicht, aktuelle Herausforderungen wie z.B. die Zielgruppenorientierung zu bewältigen sowie Rationalisierungspotentiale und Gestaltungsspielräume zu identifizieren und diese als Wettbewerbschancen zu nutzen. XML repräsentiert im engeren Sinne eine Metasprache zur Definition von Auszeichnungssprachen. Hier wird XML im weiteren Sinne als Oberbegriff für eine Reihe von Technologien verstanden, die im Zusammenhang mit XML verwendet werden. Der nutzenstiftende Einsatz von XML in der Umweltinformatik sowie im Umweltdokumentenmanagement generell ist bereits dokumentiert [West00, S. 110]: Die Eignung beruht v.a. auf den Eigenschaften der Wiederverwendbarkeit, Austauschbarkeit und Trennung zwischen Inhalt, Struktur und Darstellung. XML ist systemneutral, plattformübergreifend, erweiterbar, für Internetanwendungen angepaßt und strukturorientiert. XML-Dokumente sind maschinell validierbar und zugleich für Menschen lesbar. Daneben wird XML im Vergleich zu anderen Datenformaten speziell für die Umweltberichterstattung von Unternehmen als vorteilhaft betrachtet [ArChGö00, S. 94].

Anknüpfend an die neueren Entwicklungstendenzen in der Geschäfts- und Umweltberichterstattung sowie aufbauend auf den generellen Eignungskriterien von XML für das Umweltdokumentenmanagement wird der Nutzen von XML für die integrierte Geschäfts- und Umweltberichterstattung präzisiert [LeIsRe01, S. 60], und zwar in Zuordnung zu den vier Kernprozessen: Erstellung, Verwaltung, Verteilung und Präsentation von Geschäfts- und Umweltberichten (Abb. 2). Als konkrete Anwendung wird hier die Offenlegung von Geschäftsinformation in Umweltberichte gewählt (Stufe 1b). Im Zentrum steht dabei die Entwicklung einer umfassenden Dokument Type Definition (DTD), wobei für eine allgemeingültige DTD im Sinne einer Environmental Markup Language (EML): (1) relevante Strukturvorschläge, (2) einschlägige Leitfäden, (3) bisherige Ausprägungen von Umweltberichten sowie (4) Anforderungen von Zielgruppen explizit und systematisch einbezogen werden sollten. Am Lehrstuhl BiOR wurde eine solche XMLbasierte DTD für Umweltberichte entwickelt [Reit00; LeIsRe01]. Deren Entwicklung orientiert sich an einem umfassenden Vorgehensmodell und ist in vier Phasen gegliedert [Schr97, S. 141]:

Phase 1: Die Definition des Ziels besteht in der Entwicklung einer DTD für XMLbasierte Umweltberichte. Die Anforderungen der in Deutschland relevanten Strukturvorschläge sollten explizit berücksichtigt werden. Die DTD sollte umfassend, integrativ und flexibel sein sowie der Standardisierung dienen. 


\begin{tabular}{|l|l|l|l|}
\hline Kernprozeß & $\begin{array}{l}\text { Informationsmanagement- } \\
\text { bezogene Herausforderung }\end{array}$ & $\begin{array}{l}\text { Nutzen von XML für die } \\
\text { Berichterstattung }\end{array}$ & XML-Technologie \\
\hline Erstellung & $\begin{array}{l}\text { - hohe Dokumentenbindung } \\
\text { - notwendige Arbeitsteilung } \\
\text { - Ansätze zur Standardisierung } \\
\text { - Anstieg der Berichtszwecke }\end{array}$ & $\begin{array}{l}\text { - standardisierbare Struktur } \\
\text { - automatisierte Erstellung } \\
\text { - mächtige Verknüpfbarkeit }\end{array}$ & $\begin{array}{l}\text { - DTD, XMLSchema } \\
- \text { XLink, XPointer, XPath } \\
\text { - XML-Parser (DOM/SAX) }\end{array}$ \\
\hline Verwaltung & $\begin{array}{l}\text { - hohe Dokumentenbindung } \\
\text { - notwendige Arbeitsteilung }\end{array}$ & $\begin{array}{l}\text { - Strukturiertheit der Daten } \\
\text { - Kennzeichnung durch } \\
\text { Metadaten }\end{array}$ & $\begin{array}{l}\text { - XML-Datenbanken } \\
\text { - RDF }\end{array}$ \\
\hline Verteilung & $\begin{array}{l}\text { - Forderung nach Zielgruppen- } \\
\text { orientierung } \\
\text { - mediale Verfügbarkeit }\end{array}$ & $\begin{array}{l}\text { - integrierte Umwelt- } \\
\text { kommunikation } \\
- \text { Verteilung auf } \\
\text { verschiedenen Medien }\end{array}$ & $\begin{array}{l}\text { - XSL FO } \\
\text { - Formate, die XML-Daten } \\
\text { verarbeiten }\end{array}$ \\
\hline - CDF
\end{tabular}

Abbildung 2: Nutzen von XML für die Prozesse der integrierten Geschäfts- und Umweltberichterstattung

Phase 2: Die potentiellen semantischen Komponenten werden aus: (1) den herangezogenen Strukturvorschlägen und (2) einschlägigen Leitfäden, (3) den analysierten Dokumentinstanzen sowie (4) den Anforderungen der Zielgruppen als anvisierte Nutzer ermittelt. Die Strukturvorschläge bilden die hierbei zu beachtenden Restriktionen. Sie umfassen: EMAS II [EU01], die DIN EN ISO 14001 „Umweltmanagementsysteme“ [DIN96], die DIN 33922 „Umweltberichte für die Öffentlichkeit“ [DIN97], den Leitfaden „Umweltberichte - Umwelterklärungen. Hinweise zur Erstellung und Verbreitung“ [futu94] sowie die internationalen Empfehlungen „Company Environmental Reporting“ [UNEP94]. Anhand von Dokumentinstanzen wurden semantische und auch logische Komponenten wie z.B. Überschrift, Absatz, Graphik und Kennzahl identifiziert. Ferner wurden Analysen zu inhaltlichen und formalen Anforderungen der Zielgruppen von Umweltberichten einbezogen [IsLe01a, S. 106-107].

Phase 3: Auf der Basis der identifizierten potentiellen semantischen Komponenten wurden die relevanten semantischen Komponenten durch ein Auswahlverfahren abgeleitet. Dabei wurden die Restriktionen, Dokumentinstanzen und Anforderungen der Nutzer gemäß der abgestuften Relevanz sukzessive auf Konsistenz, Redundanz oder Ablehnung geprüft. Nach dem Abgleich ergaben sich insgesamt 119 semantische Komponenten (Abb. 3). Bei jeder Komponente ist angegeben, ob sie vorhanden sein muß (required) oder bei Bedarf integriert werden kann (optional) und auf welcher Grundlage die Komponente basiert. Neben semantischen wurden logische Komponenten aus den Dokumentinstanzen übernommen. 


\begin{tabular}{|c|c|c|c|l|}
\hline Nr. & \multicolumn{2}{|l|}{ description } & r/o & source \\
\hline 1 & \multicolumn{2}{|l|}{ preface } & 0 & instances \\
\hline 2 & \multicolumn{2}{|l|}{ organisation } & $\mathrm{r}$ & EMAS II, A III, 3.2 \\
\hline 3 & \multicolumn{2}{|l|}{ organisation description } & 0 & instances \\
\hline 4 & & relationship to parent organisation & 0 & EMAS II, A III, 3.2 \\
\hline 5 & & sites & 0 & future 6.1, I \\
\hline 6 & & number of employees & 0 & future 6.1, I \\
\hline 7 & & turnover & 0 & future 6.1, I \\
\hline 8 & & activities & $\mathrm{r}$ & EMAS II, A III, 3.2 \\
\hline 9 & & products & $\mathrm{r}$ & EMAS II, A III, 3.2 \\
\hline 10 & & new ecologically friendly products & 0 & users \\
\hline 11 & & services & $\mathrm{r}$ & EMAS II, A III, 3.2 \\
\hline 12 & & production processes & 0 & future 6.1, I \\
\hline 13 & & plants & 0 & DIN 33922, 5.1 \\
\hline 14 & \multicolumn{2}{|l|}{ environmental sector trends } & 0 & future 6.1, IV \\
\hline 15 & \multicolumn{2}{|l|}{ state of environmental technology } & 0 & users \\
\hline 16 & \multicolumn{2}{|l|}{ current topics/projects } & 0 & instances \\
\hline 17 & environmental policy & $\mathrm{r}$ & EMAS II, A III, 3.2 \\
\hline 18 & \multicolumn{2}{|l|}{ environmental guidelines } & 0 & future 6.1, II \\
\hline$\cdots$ & $\cdots$ & $\cdots$ & $\cdots$ & $\cdots$ \\
\hline
\end{tabular}

Abbildung 3: Auszug aus den relevanten 119 semantischen Komponenten

Phase 4: Mit den ausgewählten Komponenten wurde das Dokumenttypmodell entworfen. Dazu wurden die ausgewählten Komponenten in einer XML-typischen Hierarchie strukturiert. Zur Ausschöpfung der XML-spezifischen Vorzüge wurde eine Mischform aus Struktur- und Inhaltsorientierung gewählt (Abb. 4).
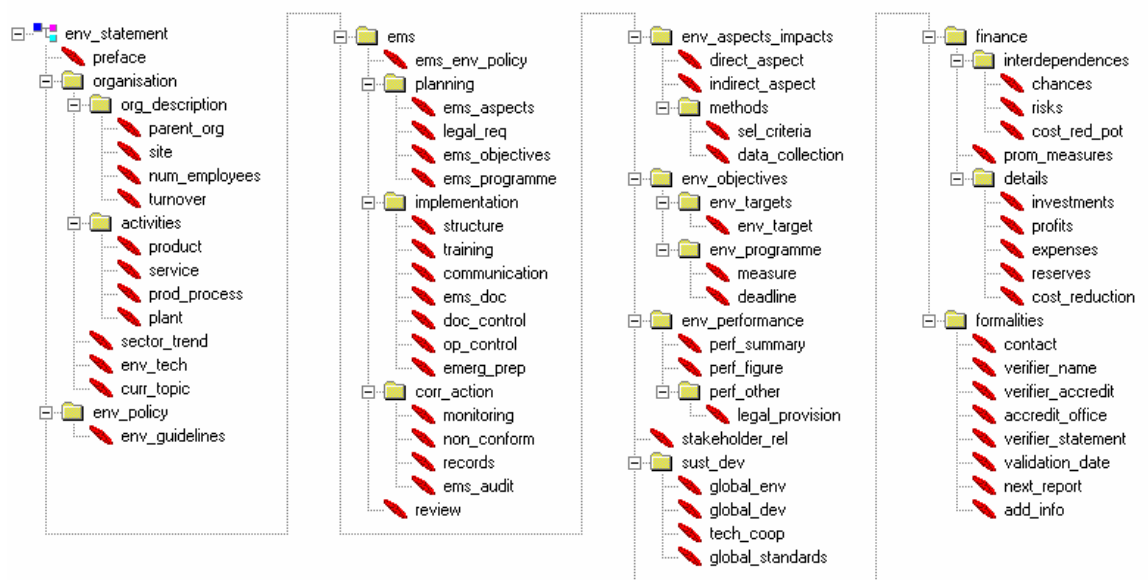

Abbildung 4: Hierarchie eines XML-basierten Umweltberichts mit Geschäftsinformation

Das Dokumenttypmodell wurde XML-konform notiert (Abb. 5) und in Form einer XML-basierten DTD implementiert. Die erste Strukturebene der DTD umfaßt 12 Elemente. 


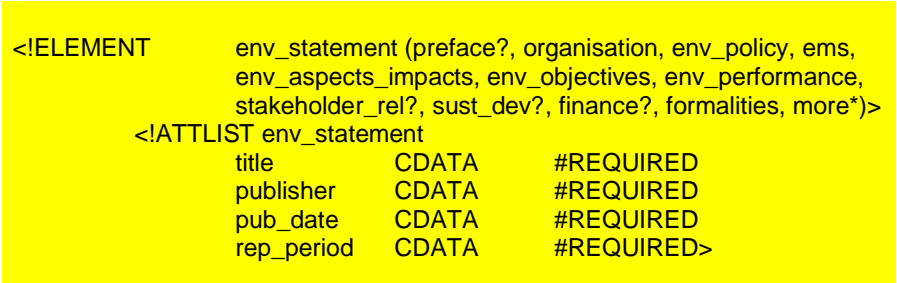

Abbildung 5: Auszug der XML-basierten DTD für Umweltberichte

Die auf Basis dieser DTD erstellten Umweltberichte mit offengelegter Geschäftsinformation (,finance“ aus Abb. 4) können objektiv verglichen werden. Das erhöht die Aussagekraft für Unternehmen und den Nutzen für die Zielgruppen. Der Einsatz von XML unterstützt insgesamt eine durchgängige, schnittstellenarme und medienbruchfreie Erstellung von Umweltberichten [LeIsRe01, S. 63]. Eine umfassende XML-DTD hat dabei Leitfadencharakter und bietet eine Hilfe zur Standardisierung von Umweltberichten.

\section{Schritt zur Nachhaltigkeitsberichterstattung}

Vieles spricht für eine integrierte Geschäfts- und Umweltberichterstattung als wichtiger Schritt zur Nachhaltigkeitsberichterstattung: In der sich formierenden Informationsgesellschaft und unter den Bedingungen eines globalen Wettbewerbs scheint eine aktive und informative Kommunikation mit den verschiedenen Zielgruppen über die ökonomische und ökologische Leistung eine wichtige Aufgabe der Unternehmen zu sein [FiSc00]. Das belegen: (1) die Hinweise der verschiedenen Initiativen zur Nachhaltigkeitsberichterstattung [GRI00; IÖW00], (2) die Konzepte zu einem „,nachhaltigen Unternehmen“ [FiCl98] und „Sustainable Management" [IsWa99b, S. 75] ebenso wie die (3) EU-Initiativen zur integrierten Berichterstattung [EU00; EU99]. Im Zuge der Ausweitung der Geschäftstätigkeit nehmen expandierende Unternehmen zur Deckung ihres Kapitalbedarfs vermehrt internationale Kapitalmärkte in Anspruch. Die Bereitschaft der Investoren, Aktien zu erwerben und zu halten, hängt wesentlich von dem Vertrauen ab, das sie den Unternehmen entgegenbringen. Vertrauen erzeugen Unternehmen bei Investoren und Multiplikatoren u.a. durch eine aktive und informative Berichterstattung über die wirtschaftliche Lage und Entwicklung der Geschäftstätigkeit. Im Blick auf das in Unternehmen gereifte Bekenntnis zum Ziel einer nachhaltigen Entwicklung kommt zuzüglich zu den gesetzlichen Rechenschafts- und Investor-Relations-Instrumenten der Umweltberichterstattung eine wachsende Bedeutung zu. Eine integrierte Berichterstattung bietet dabei Wettbewerbschancen, und das Internet eröffnet wirksame Unterstützungspotentiale. 
Aus IKT-spezifischer Sicht werden die internetbasierten Unterstützungspotentiale die Geschäfts- und Umweltberichterstattung von Unternehmen von Grund auf verändern [IsLeMM01, S. 105]: Das Internet repräsentiert mehr als ein Mittel zur aktuellen, interaktiven und hypermedialen Präsentation von integrierten Nachhaltigkeits- oder separaten, durch Hyperlinks verknüpften Geschäfts- und Umweltberichten. Es repräsentiert auch mehr als einen Distributionskanal mit einer großen Reichweite und einer 24h-Verfügbarkeit. Es ermöglicht eine integrierte Berichterstattung, in der sämtliche Prozesse unterstützt werden: Geschäfts- und Umweltdaten werden dezentral erfaßt, zu relevanter Information verdichtet, zweckentsprechend systematisiert, zu hypermedialen Berichten aufbereitet, zielgruppenorientiert zugänglich und medienübergreifend verfügbar gemacht. Die internetbasierten Unterstützungspotentiale drängen mit Macht in sämtliche Prozesse der Berichterstattung hinein, von der effizienten Erstellung und Verwaltung bis hin zur zielgruppenorientierten Verteilung und ansprechenden Präsentation. Mit ihrer konsequenten Ausschöpfung geht eine Neugestaltung einher, sowohl bei den Prozessen der Geschäfts- und Umweltberichterstattung in Unternehmen als auch beim Inhalt und der Nutzung von Berichten durch die Zielgruppen selbst.

Ungeachtet der Unterstützungspotentiale vermag der Einsatz des Internet alleine weder die traditionellen Formen der Geschäfts- und Umweltberichterstattung komplett zu ersetzen noch die anderen Möglichkeiten wie z.B. Road-Shows, Hauptversammlungen, Pressekonferenzen, Unternehmenszeitung usw. zu verdrängen. Eine integrierte Geschäfts- und Umweltberichterstattung auf der einheitlichen und medienübergreifenden technischen Basis des Internet sollte weder als universell geeignetes Instrument überfrachtet noch die Qualität der Berichterstattung mit der ökonomischen und ökologischen Leistung des Unternehmens einfach gleichgesetzt werden. Vielmehr wird hier einerseits für die Beachtung der technischen Standards und andererseits für eine nutzenstiftende Ergänzung und ggf. teilweise Substitution traditioneller Formen argumentiert; zum einen aus Gründen einer rationellen Erstellung, Verwaltung und Verteilung und zum anderen aus Gründen sinnvoller Integration, erhöhter Aussagekraft, mehrwertstiftender Präsentation und erweiterter Kommunikation durch hypermediale Darstellung, Interaktivität, Dialog und Zielgruppenorientierung.

Eine seriöse Analyse einer integrierten Geschäfts- und Umweltberichterstattung auf der Basis des Internet sollte auch mögliche Nachteile, nicht nur die Vorzüge in Form von Unterstützungspotentialen herausstellen. Die Nachteile beziehen sich v.a. auf drei medienbedingte Grenzen [IsWab99b, S. 76]: (1) Die Reichweite hängt von einer geeigneten IKT-Infrastruktur ab, sowohl bei den berichterstattenden Unternehmen als auch bei den Zielgruppen. (2) Die ökologischen Belastungen durch den Interneteinsatz sollten entlang des gesamten ökologischen Lebenszyklusses - d.h. von der Erstellung, Verwaltung und Verteilung bis zur Nutzung bei den Zielgruppen - berücksichtigt werden. (3) Der Nutzen einer integrierten Berichterstattung auf der Basis des Internet wird maßgeblich von der Akzeptanz der Zielgruppen beeinflußt und durch den wahrgenommenen Mehrwert entweder eher 
gefördert oder aber eher abgelehnt. Diese drei Grenzen gilt es zu beachten. Gleichwohl kann eine integrierte Geschäfts- und Umweltberichterstattung auf der Basis des Internet dazu beitragen, Wettbewerbsvorteile für Unternehmen, Anforderungen von Zielgruppen mit neuen Formen der Geschäfts- und Umweltkommunikation und Entlastungen der Natur zu verbinden. Nicht nur deshalb sollten die Möglichkeiten im Dienste einer nachhaltigen Entwicklung beachtet, im Belastungstest der Praxis auf ihre Tauglichkeit untersucht und die Unterstützungspotentiale ausgeschöpft werden.

\section{Literatur}

[Anto00] Antonoff, A.: Anleger sehen im Internet oft schwarz. In: Die Welt online vom 24.11.2000 (Finanzen). In: http://www.welt.de, Abruf am 2001-03-06

[ArChGö00] Arndt, H-K; Christ, M.; Görsch, D.: XML als Metasprache zum Entwurf einer standardisierten Beschreibung von Umweltdaten. In: Arndt, H.-K.; Günther, O. (Hrsg.): Environmental Markup Language (EML). Metropolis, Marburg 2000, S. 9-29

[BaKi97] Baetge, J.; Kirchhoff, K.R.: Der Geschäftsbericht: Die Visitenkarte des Unternehmens. Ueberreuter, Wien 1997

[DAI00] Deutsches Aktieninstitut (DAI): Investor Relations im Internet. Internet-Angebote börsennotierter Unternehmen im Vergleich. DAI, Frankfurt am Main 2000

[Dald99] Daldrup, H.: Publizität umweltschutzbezogener Informationen in Geschäftsberichten. In: Die Wirtschaftsprüfung 52 (1999) 18, S. 734-748

[DIN96] Deutsches Institut für Normung e.V. (DIN): DIN EN ISO 14001. Umweltmanagementsysteme. Spezifikation mit Anleitung zur Anwendung. Beuth, Berlin 1996

[DIN97] Deutsches Institut für Normung e.V. (DIN): DIN 33922. Leitfaden Umweltberichte für die Öffentlichkeit. Beuth, Berlin 1997

[EU00] Kommission der Europäischen Gemeinschaften (KOM): Rechnungslegungsstrategie der EU: Künftiges Vorgehen. Brüssel 2000

[EU01] Verordnung (EG) Nr. 761/2001 des Europäischen Parlaments und des Rates vom 19. März 2001 über die freiwillige Beteiligung von Organisationen an einem Gemeinschaftssystem für das Umweltmanagement und die Umweltbetriebsprüfung (EMAS II). In: Amtsblatt der EG L 114/1 vom 24.4.2001, Brüssel

[EU99] Kommission der Europäischen Gemeinschaften: Entwurf. Empfehlung der Kommission zur Berücksichtigung von Umweltaspekten in Jahresabschluß und Lagebericht der Unternehmen. Brüssel 1999

[FiCl98] Fichter, K.; Clausen, J. (Hrsg.): Schritte zum nachhaltigen Unternehmen. Springer, Berlin et al. 1998

[FiSc00] Fichter, K.; Schneidewind, U. (Hrsg.): Umweltschutz im globalen Wettbewerb. Springer, Berlin et al. 2000 
[futu94] future e.V. (Hrsg.): Leitfaden Umweltberichte - Umwelterklärungen. Hinweise zur Erstellung und Verbreitung. future e.V., Osnabrück 1994

[GRI00] Global Reporting Initiative (GRI): Leitfaden für Nachhaltigkeitsberichte. GRI, Boston 2000

[Grön00] Gröner, S.: Umweltberichterstattung für eine nachhaltige Entwicklung. Shaker, Aachen 2000

[Helm98] Helm, L.: Electronic Publishing. In: Müller, M.; Leven, F.-J. (Hrsg.): Shareholder Value Reporting. Ueberreuter, Wien 1998, S. 103-118

[Henk00] Henkel KGaA: Instrumente der Henkel SHE-Kommunikation. http://www.she.henkel.de/html/she_komm.htm, Abruf am 2000-12-05

[HGB01] Hamburger Geschäftsberichte GmbH: Wir bieten integrierte Konzeptionen. http://hgb.de/content/wwt/konzept.htm, Abruf am 2001-03-06

[Hütt00] Hütten, C.: Der Geschäftsbericht als Informationsinstrument. Hagemann, Düsseldorf 2000

[IÖfu00] Institut für ökologische Wirtschaftsforschung (IÖW); future e.V.: Ranking 2000. http://www.ranking-umweltberichte.de, Abruf am 2000-08-14

[IÖW00] Institut für ökologische Wirtschaftsforschung (IÖW); Institut für Markt - Umwelt Gesellschaft (IMUG) (Hrsg.): Schritte zur Nachhaltigkeitsberichterstattung. IÖW, Berlin 2000

[IsBu00] Isenmann, R.; Busch, H.: Hypermediale Umweltberichte: Funktionen und Prozesse In: Riekert, W.-F.; Tochtermann, K. (Hrsg.): Hypermedia im Umweltschutz. Metropolis, Marburg 2000, S. 134-146

[Isen01] Isenmann, R.: Hypermediale Umweltberichte von Unternehmen: Bestandsaufnahme - Empirische Befunde - Klassifikation. In: Tochtermann, K.; Riekert, W.-F. (Hrsg.): Neue Methoden für das Wissensmanagement im Umweltbereich. Metropolis, Marburg 2001, S. 33-45

[IsLe01a] Isenmann, R.; Lenz, C.: Customised Environmental Reports by Internet-based Push- and Pull-Technologies. In: Eco-Management and Auditing 8 (2001) 2, S. 100-110

[IsLe01b] Isenmann, R.; Lenz, C.: System of Technical Benefits Using the Internet for Corporate Environmental Reporting. In: ERP Environment (Ed.): The 2000 Business Strategy and the Environment Conference. ERP, Shipley (UK), 2000, S. 203-210

[IsLeMM01] Isenmann, R.; Lenz, C.; Müller-Merbach, H.: Betriebliche Umweltberichterstattung im Internet. In: Praxis der Wirtschaftsinformatik 38 (2001) 218, S. 97-107

[IsLeSc01] Isenmann, R.; Lenz, C.; Schweren, C.: Interneteinsatz zur integrierten Geschäftsund Umweltberichterstattung. In: Treibert, R. (Hrsg.): Betriebliche Informationssysteme für Umwelt, Qualität und Sicherheit. Metropolis, Marburg 2001, S. 47-61

[IsScMM01] Isenmann, R.; Schweren, C.; Müller-Merbach H.: Einsatz des Internet zur Umweltberichterstattung von Unternehmen. Universität Kaiserslautern. BiOR 2001 
[IsWa99a] Isenmann, R.; Warkotsch, N.: Internetbasierte Umweltberichterstattung. In: Das Wirtschaftsstudium 28 (1999) 11, S. 1509-1517

[IsWa99b] Isenmann, R.; Warkotsch, N.: Unterstützungspotentiale internetbasierter Umweltberichterstattung (II). In: Umweltwirtschaftsforum 7 (1999) 3, S. $72-76$

[Jone99] Jones K.: Study on Environmental Reporting by Companies. Commisioned by the European Commission (EU). University of Sunderland (UK) 1999

[KPMG99] KPMG Environmental Consulting (Hrsg.): KPMG International Survey of Environmental Reporting 1999. Reynen, Amstelveen 1999

[KrGr99] Krumnow, J.; Gramlich, L. (Hrsg.): Gabler-Lexikon: Bank - Börse - Finanzierung. Gabler, Wiesbaden 1999

[LaAhDa01] Lange, C.; Ahsen, von A.; Daldrup, H.: Umweltschutz-Reporting. Oldenbourg, München 2001

[LeIsRe01] Lenz, C.; Isenmann, R.; Reitz, C.: Zielgruppenorientierte Umweltberichterstattung von Unternehmen mit XML. In: Riekert, W.-F.; Tochtermann, K. (Hrsg.): Neue Methoden für das Wissensmanagement im Umweltbereich. Metropolis, Marburg 2001, S. 57-69

[LoFi99] Loew, T.; Fichter, K.: Umweltberichterstattung in Deutschland und Europa. IÖWSchriftenreihe Nr. 138/99. IÖW, Berlin 1999

[psyc00] psychonomics. Studien 2000. Investor Relations - State of the Art im Internet 2000. In: http//www.psychonomics.de/6074/investorrelation.htm, Abruf am 2001-02-23

[Reit00] Reitz, C.: Internetbasierte Umweltberichterstattung mit XML. Universität Kaiserslautern. Diplomarbeit BiOR 2000

[Schr97] Schraml, T.: Operationalisierung der ökologiebezogenen Berichterstattung aus Sicht des Informationsmanagements. Dissertation TU Dresden 1997

[Schu95] Schulz, T.M.: Ökologieorientierte Berichterstattung von Unternehmen. Haupt, Bern et al. 1995

[StScLe97] Steven, M.; Schwarz, E.; Letmathe, P.: Umweltberichterstattung und Umwelterklärung nach der EG-Öko-Audit-Verordnung. Springer, Berlin et al. 1997

[Stur00] Sturm, A.: Performance Measurement und Environmental Performance Measurement. Dissertation TU Dresden 2000

[Uka86] Uka, W.: Umweltberichterstattung der Großunternehmen. IIUG Report 86-13. Wissenschaftszentrum Berlin für Sozialforschung 1986

[UNEP94] United Nations Environment Programme Industry and Environment (UNEP): Company Environmental Reporting. Technical Report 24. UNEP, Paris 1994

[West00] Westbomke, J. et al.: XML und Umweltdokumentmanagement. In: Riekert, W.-F.; Tochtermann, K. (Hrsg.): Hypermedia im Umweltschutz. Metropolis, Marburg 2000, S. $105-116$ 